UDC 347

LBC 67.404

\title{
LEGAL REGIME OF THE RESULTS OF INTELLECTUAL ACTIVITY OF WORKERS OF ORGANIZATIONS IN THE RUSSIAN FEDERATION
}

\author{
Olga V. Nikulina \\ Volgograd Institute of Management - the Branch of the Russian Academy of National Economy \\ and Public Administration under the President of the Russian Federation, Volgograd, Russian Federation
}

Introduction: the relevance of the topic is determined by the need to develop recommendations for the application of existing legislation, as well as the adoption of legal norms that are in line with current realities and take into account the development trends of relations. The development of institutions of official works, inventions in the Russian Federation does not stop to this day. There is a need for solving new tasks, specifying and detailing the norms of law. Legal regulation in the sphere of creation and use of official results of intellectual activity is not balanced, the lack of uniformity in law enforcement practice causes many problems. The purpose of the research is to disclose the concept of an official result of an activity, as well as the grounds for the emergence of a legal regime for the service result of intellectual activity. Methods are applied in aggregate methods of scientific cognition, among which the main ones are the methods of historicism, system, analysis and comparative-legal. Results: the necessity of providing legal protection to the project of the service object of intellectual property is justified. Granting legal protection to the "project" of the service result of intellectual activity and securing the rights of the employee and employer would enable them to meet the interests of both parties. This will help overcome this gap in the legal regulation of legal relationships to create and use official creative result. The definition of the service result of intellectual activity is proposed. The service result of intellectual activity should be defined as the object of intellectual rights, protected by law, that meets the criterion of applicability in the sphere of activity of the employer, as created by the performance of work (service) duties, as well as the specific task of the employer by the author-participant in labor (service) relations. Conclusions: it is necessary to build norms on the performance of intellectual activity in a single system. The Civil Code of the Russian Federation should contain general provisions that fix the concept of an official object of intellectual property.

Key words: service work, service invention, service selection achievement, service secret of production, exclusive right, patent, examination of applications for invention, contract of author's order.

УДК 347

ББК 67.404

\section{ПРАВОВОЙ РЕЖИМ РЕЗУЛЬТАТОВ ИНТЕЛЛЕКТУАЛЬНОЙ ДЕЯТЕЛЬНОСТИ РАБОТНИКОВ ОРГАНИЗАЦИЙ В РОССИЙСКОЙ ФЕДЕРАЦИИ}

\author{
Ольга Владимировна Никулина \\ Волгоградский институт управления - филиал Российской академии народного хозяйства \\ и государственной службы при Президенте РФ, г. Волгоград, Российская Федерация
}

Введение: актуальность темы определяется необходимостью разработки рекомендаций по применению действующего законодательства, а также принятия правовых норм, соответствующих современным реалиям и учитывающих тенденции развития отношений. Развитие институтов служебных произведений, изобретений в РФ не прекращается по сей день. Существует потребность в решении новых задач, конкретизации и детализации норм права. Правовое регулирование в сфере создания и использования служебных результатов интеллектуальной деятельности не сбалансировано, отсутствие единообразия в правопримени- 
тельной практике порождает множество проблем. Цель исследования - раскрыть понятие служебного результата деятельности, а также основания возникновения правового режима служебного результата интеллектуальной деятельности. Методы: применены в совокупности методы научного познания, среди которых основные - методы историзма, системности, анализа и сравнительно-правовой. Результаты: обоснована необходимость предоставления правовой охраны проекту служебного объекта интеллектуальной собственности. Предоставление правовой охраны «проекту» служебного результата интеллектуальной деятельности и закрепление прав работника и работодателя позволили бы соблюсти интересы обеих сторон. Это поможет преодолеть указанный пробел в правовом регулировании правоотношений по созданию и использованию служебного творческого результата. Предложено определение служебного результата интеллектуальной деятельности. Служебный результат интеллектуальной деятельности следует определить как созданный в ходе выполнения трудовых (служебных) обязанностей, а также конкретного задания работодателя автором - участником трудовых (служебных) отношений, охраняемый правом объект интеллектуальных прав, отвечающий критерию применимости в сфере деятельности работодателя. Выводы: необходимо выстроить в единую систему нормы о служебных результатах интеллектуальной деятельности. ГК РФ должен содержать общие положения, закрепляющие понятие служебного объекта интеллектуальной собственности.

Ключевые слова: служебное произведение, служебное изобретение, служебное селекционное достижение, служебный секрет производства, исключительное право, патент, экспертиза заявок на изобретение, договор авторского заказа.

UDK 347

BBK 67.404

\title{
RECHTLICHER SCHUTZ VON PRODUKTEN DER INTELLEKTUELLEN TÄTIGKEIT DER BEDIENSTETEN IN DER RUSSISCHEN FÖDERATION
}

\author{
Olga W. Nikulina \\ Russische Akademie der Volkswirtschaft und öffentliche Verwaltung beim Präsidenten \\ der Russischen Föderation, Wolgograd, Russischen Föderation
}

Einführung: die Relevanz des Themas wird durch die Notwendigkeit bestimmt, Empfehlungen für die Anwendung der bestehenden Rechtsvorschriften zu entwickeln sowie Rechtsvorschriften zu beschließen, die modernen Gegebenheiten entsprechen und gegenwärtige Trends in der Entwicklung der Rechtsverhältnisse berücksichtigen. Die Entwicklung von Institutionen, deren Tätigkeit auf Schaffung von amtlichen Produkten und Erfindungen gerichtet ist, wird in der Russischen Föderation nicht für einen Tag unterbrochen. Es ist nötig, neue Herausforderungen zu bewältigen, Rechtsnormen zu konkretisieren und spezifizieren.

Gesetzliche Regelung im Bereich der Schaffung und Verwendung von dienstlichen (amtlichen) Produkten ist nicht genügend ausballanciert, es gibt einen Mangel an Einheitlichkeit in der Rechtspraxis bei der Behandlung von vielen Problemen. Der Zweck der Forschung ist es, den Begriff der in verschiedenen Ämtern entstandenen dienstlichen Produkte zu klären sowie die Entstehung der rechtlichen Regelung von Produkten der intellektuellen Tätigkeit zu begründen. Methoden der wissenschaftlichen Erkenntnisse werden in Kombination verwendet, darunter sind als die wichtigsten folgende zu nennen: historisches Herangehen, systemhafte Analyse und Rechtsvergleichung. Ergebnisse: es ist die Notwendigkeit begründet, dienstliche Produkte des geistigen Eigentums rechtlich zu schützen, die Verrechtlichung der Verhältnisse der Arbeitnehmer und der Aebeitgeber in diesem Bereich würden beiderseitige Interessen wahrnehmen. Dies wird dazu beitragen, diese Lücke in der gesetzlichen Regelung der Rechtsbeziehungen auf dem Gebiet von Schaffung und Anwendung vonr kreativen dienstlichen Produkten zu füllen. Es wird ebenfalls eine Definition des dienstlichen Produktes der intellektuellen Tätigkeit gegeben, das verstanden wird als Produkt, das von dem Mitarbeiter, dem Schöpfer, Autor und einem der Trägern von Arbeits- oder Dienstverhältnissen, im Laufe der Erledigung seiner Amtsflichten sowie eines konkreten Auftrages des Arbeitsgebers geschaffen wird, das als Objekt der Rechtsverhältnisse rechtlich geschützt wird und das den Kriterien der Anwendbarkeit in der Tätigkeit des Arbeitsgebers entspricht.

Schlussfolgerungen: es ist notwendig, Rechtsnormen für Produkte der intellektuellen Tätigkeit als ein einheitliches System zu erarbeiten. Das BGB der RF sollte allgemeine Bestimmungen enthalten, die den Begriff "dienstliches Oblekt des geistigen Eigentums" verankern würden. 
Stichworte: dienstliches Produkt, dienstliche Erfindung, dienstliche selektive Leistung, Dienstgeheimnis, ausschließliches Recht, Patent, Prüfung von Anträgen auf Erfindungen, Vertrag des Autorsauftrages.

\section{Einführung}

Eine große Anzahl von Produkten der intellektuellen Tätigkeit wird als dienstliche oder amtliche Werke geschaffen. Die Entwicklung der Institutionen der dienstlichen Produkte und Erfindungen hört nie auf, weil eine Notwendigkeit besteht, neue Herausforderungen zu bewältigen, Rechtsnormen $\mathrm{zu}$ konkretisieren und $\mathrm{zu}$ specifizieren. Gesetzliche Regelung im Bereich der Erarbeitung und Verwendung von dienstlichen Produkten der intellektuellen Tätigkeit ist unzureichend ausballanciert. Es gibt keine Einheitlichkeit in der Rechtsanwendungspraxis, was zu vielen Problemen führt.

\section{Dienstliches Objekt \\ der intellektuellen Rechte als Ergebnis der Arbeitsleistung eines Mitarbeiters}

Vierter Teil des BGB nennt folgende dienstliche Produkte der intellektuellen Tätigkeit: dienstliches Produkt (Art. 1295); dienstliche Aausführung (Art. 1320); dienstliche Erfindung, dienstliches Nutzungsmodell (oder Gebrauchsmuster), dienstliches Produktionsmuster (Art. 1370); Selektionsleistung (Art. 1430); dienstliche Topologie von integrierten Chips (Art. 1461); dienstliches Produktionsgeheimnis (Art. 1470).

Unter einem dienstlichen Produkt ist nach Absatz 1 des Artikels 1295 des BGB ein Produkt $\mathrm{zu}$ verstehen, das von dem Mitarbeiter im Rahmen der Ausführung seiner Dienstpflichten geschaffen worden ist. Wir können sagen, Diensprodukte seien nur jene Produkte, deren Schaffung sich dank Dienstpflichten des Mitarbeiters ergeben hat.

Unter solchen Produkten, die von einem Mitarbeiter in einer Diensstelle geschaffen werden, sind Computerprogramme auszusondern. Das Computerprogramm, das in einer objektiven Form die Gesamtsumme von Daten und Anordnungen darstellt, ist für Betrieb von Computern und anderen Rechenanlagen nötig, ein bestimmtes Ergebnis zu erreichen. Der Begriff umfasst auch: a) Vorbereitungsmaterialien für Entwicklung des Computerprogramms; b) die durch das Programm produzierten audio-visuellen Programmkomponenten.

Solche Produkte können im Rahmen der Erfüllung eines Arbeitsvertrages oder eines Auftrags, bestimmte Forschungen durchtzführen, Konstruktionsarbeiten und technologische Arbeiten auszuführen (Art. 1297 des BGB). In diesem Fall gelten sie als dieenstliche Produkte der intellektuellen Tätigkeit nicht.

Gemäß dem Art. 1350 des BGB wird unter Erfindung eine technische Lösung in einem bestimmten Bereich verstanden, die zu einem Produkt (Gerät, Substanz) oder Verfahren (Verfahren, bestimmte Handlungen an einem materiellen Objekt zu vollziehen) gehört sowie die Verwendung von Produkt oder Verfahren zu einem bestimmten Zweck betrifft.

Eine Erfindung wird rechtlich geschützt, wenn es dabei geht: um eine ganz neue Erfindung (sie ist aus dem gegenwärtigen Stand der technischen Entwicklung nicht bekannt), um ein bestimmtes Erfindungsniveau (der Fachmann leitet die Erfindung nicht immer von dem Stand der technischen Entwicklung ab) und um industrielle Anwendbarkeit.

Gemäß dem Abs. 1 des Art. 1370 des BGB soll man unter einer dienstlichen Erfindung solche verstehen, die von einem Mitarbeiter im Ergebnis der Erfüllung seiner arbeitsvertraglichen Pflichten sowie einer konkreten Aufgabe des Arbeitgebers entsteht.

Die Tatsache der Schaffung dieses kreativen Produktes von dem Autor während der Gültigkeit seines Arbeitsvertrages gibt noch keinen Grund, dieses Produkt als dienstliches Werk der intellektuellen Tätigkeit zu bewerten. Für Entstehung der rechtlichen Regelung der dienstlichen Erfindungen braucht man das Vorhandensein folgender Kette: Ursache - Folge.

Russische Rechtsvorschriften enthalten folgende erforderliche Umstände: Der Autor soll in einem Arbeitnehmerverhältnis stehen, die Erfindung ist vomAutor im Rahmen der Erfüllung seiner arbeitsvertraglichen Pflichten oder als eine bestimmte Aufgabe des Arbeitgebers zu entwickeln oder zu schaffen. Doch die Frage nach dem Zeitpunkt der Entstehung des Produktes der 
intellektuellen Tätigkeit ist in der juristischen Literatur noch strittig.

Gemäß den Vorschriften des BGB kann der

Zeitpunkt der Entstehung einer dienstlichen Erfindung in Betracht gezogen werden, wenn das Produkt der schöpferischen Tätigkeit eines Arbeitnehmers solche Chrakteristiken erwirbt wie Neuartigkeit, Erfindungsniveau und gewerbliche Anwendbarkeit.

Man muss sagen, dass nicht alle qualifizierenden Merkmale der Erfindung, die im allgemeinen Patentrecht enthalten sind, die Entstehung der Rechtsstellung für eine dienstliche Erfindung beeinflussen. Zum Beispiel kann die Patentfähigkeit aus rechtlicher Sicht keine Bedeutung haben, weil Rechtsfolgen für das Produkt der intellektuellen Tätigkeit für die vertraglichen Parteien nur durch das Gesetz zu wirken beginnen, unabhängig von der Patentfähigkeit eines solchen Produktes.

S.I. Krupko ist zum Beispiel der Meinung, dass der Zeitpunkt der Erfindung "immer dem Zeitpunkt vorangeht, in dem der Patenpschutz der Erfindung gültig wird" [5]. Das Patent ist in diesem Fall nicht eine einzig mögliche Form des rechtlichen Schutzes einer Erfindung des Mitarbeiters.

$\mathrm{Zu}$ den Erfindungen können nicht gezählt werden: wissenschaftliche Theorien; mathematische Modelle; Computerprogramme; Lösungen, durch die Informationen und andere Objekte (die im Abs. 5 des Art. 1350 des BGB angeführt sind) zur Verfügung gestellt werden. Diese Liste ist offen.

Als dienstliche Produkte gelten auch freie Erfindungen nicht. Laut dem Abs. 5 des Art. 1370 des BGB gehören zu den dienstlichen Werken folgende nicht: eine Erfindung, ein Gebrauchsmuster oder Produktionsmuster, die von dem Mitarbeiter mit Geld, technischen oder anderen Sachwerten des Arbeitgebers entwickelt sind, die aber nicht mit der Leistung seiner beruflichen Pflichten oder einer bestimmten Aufgabe des Arbeitgebers verbunden sind Das sind s.g. ursprünglich freie Erfindungen. Die gleiche Bestimmung wird in der russischen Gesetzgebung in Bezug auf andere Produkte der intellektuellen Tätigkeit verankert.

Den Status freier Erfindungen können nur diejenigen erwerben, die zum Zeitpunkt ihrer Entstehung alle Merkmale eines dienstlichen
Objektes des intellektuellen Eigentums besitzen. Ein solches kreatives Produkt kann nur durch Gesetz in eine freie Erfindung (wenn ein Arbeitgeber z.B. ein Patent innerhalb eines bestimmten Zeitraums nicht erhält) oder durch den freiwilligen Willen des Arbeitgebers umgewandelt werden. Es ist jedoch nicht klar, in welcher Form so ein Wille zum Vorschein kommen sollte.

Außer den abgeleiteten Erfindungen können andere Produkte der intellektuellen Tätigkeit ebenfalls als dienstlich anerkannt werden, und nämlich Gebrauchsmuster und Produktionsmuster (Art. 1370 des BGB). Ein Gebrauchsmuster ist laut dem Art. 1351 des BGB eine technische gerätsbezogene Lösung. Sie soll neuartig und gewerblich anwendbar sein.

Unter einem Produktionsmuster wird in der russischen Gesetzgebung eine Lösung verstanden, die auf die äußerliche Form eines industriellen oder handwerklichen Erzeugnisses gerichtet ist. Es muss den Anforderungen der Neuartigkeit und Originalität (Art. 1352 des BGB) entsprechen. Die wesentlichen Merkmale eines Produktionsmusters sind diejenigen, die Art des Erzeugnisses bestimmen, und zwar Form, Konfiguration, Ornament, Farbkombination usw. Aus dieser Definition folgt, dass der Rechtsschutz nur dem externer Produktgestalt gewährt werden kann. Eine Ausnahme bildet dabei nur die externe durch technische Funktion bedingte Form des Objekts.

Ein weiteres dienstliches Objekt der intellektuellen Rechte ist eine Selektionsleistung. Sie muss von dem Arbeitnehmer bei der Ausübung seiner beruflichen Pflichten oder eine bestimmten Aufgabe des Arbeitgebers (Art. 1430 des BGB) geschaffen, abgeleitet oder identifiziert werden.

$\mathrm{Zu}$ den Selektionsleistungen werden Sorten von Pflanzen und Tierrassen gezählt. Die Entdeckung einer neuen Pflanze oder eines Tieres in der Natur ist auch als rechtlich zu schätzende Leistung anerkannt. In diesem Sinne gibt es einige Unterschiede in den Rechtsvorschriften, weil die Entdeckung nach der juristischen Sichtweise eine Erfindung ist.

Als dienstlich kann auch ein Produktionssgeheimnis (Know-how) sein, wenn es von einen Mitarbeiter im Zusammenhang mit der Ausübung seiner beruflichen Pflichten oder einer bestimmten Aufgabe des Arbeitgebers (Art. 1470 des BGB) entsteht.

Es sei aber betont, dass als Know-hows solche Daten (produktionsbezogene, technische, 
wirtschaftliche und andere) von Objekten des geistigen Eigentums anerkannt sind wie: a) im wissenschaftlich-technischen Bereich; b) Methoden der beruflichen Tätigkeit. Weitere Informationen sind als Dienstsgeheimnis rechtlich nicht geschützt. Allerdings kann der Arbeitgeber solche Informationen zu Geschäftsgeheimnissen zuordnen.

Nach den Rechtsvorschriften der RF ist ein Geschäftsgeheimnis ein "Privacy-Modus von Informationen, der dem Inhaber erlaubt, unter bestehenden oder potentiellen Umständen Einnahmen zu erhöhen, unnötige Kosten zu vermeiden, seine Positionen auf den Waren-, Arbeits-, und Dienstleistungsmärkten zu erhalten oder andere kommerzielle Vorteile zu gewinnen"1.

Dienstliche Topologie von integrierten Chips (Art. 1461 des BGB) wird auch von dem Autor im Zusammenhang mit der Erfüllung seiner Arbeitspflichten oder einer bestimmten Aufgabe geschaffen. Nach Art. 1448 des BGB gilt als die Topologie von integrierten Chips eine auf dem materiellen Träger fixierte räumliche oder geometrische Aufstellung von Komponenten des Chips und Verbindungen zwischen diesen Elementen. Rechtlich werden nur originelle Topologien geschützt.

\section{Dienstliches Objekt der intelektuellen Rechte als Eigentum der Organisation}

Nach geltendem Recht genießen alle oben genannten dienstlichen Produkte der intellektuellen Tätigkeit geistiges Eigentumsrecht. Unter diesen gibt es persönliche Nichtvermögensrechte und Vermögensrechte (ausschließliches Recht).

Das ausschließliche Recht gehört dem Arbeitgeber. Er kann das kreative Produkt nach seinem Ermessen verwenden, auf eigene Faust und mit allen rechtlichen Mitteln. Es liegt im Interesse des Arbeitgebers, ein dienstliches Objekt des geistigen Eigentums entstehen zu lassen, da er es für das Erreichen seiner Ziele (vor allem kommerzieller Gewinne) verwenden kann.

Organisation (der Arbeitgeber) hat auch das Recht, ein Patent auf eine Erfindung, Gebrauchsmuster oder Produktionsmuster, Industriedesign $\mathrm{zu}$ erwerben.

Auf Grundlage des Befehls von Rospatent wurde im Jahr 2011 eine Anleitung für Prüfungen von Anträgen auf Erfindungen genehmigt ${ }^{2}$. Dieses Dokument vervollkommnete die Art der methodischen Behandlung bei der Durchführung von Gutachten sowie Ansätze zur Behandlung von deren Einwänden in der Kammer der Patentstreitigkeiten.

Einen breiten Umfang von Rechten hat der Arbeitgeber in Bezug auf Produktionsgeheimnisse (Know-how). Wenn der Mitarbeiter so ein Geheimnis erfahren hat, ist er verpflichtet, die Vertraulichkeit dieser bekanntgewordenen Informationen bis zur Beendigung der Gültigkeit des ausschließlichen Rechts zu bewharen.

Strittig bleibt auch die Frage nach Möglichkeit für den Mitarbeiter, einen Entwurf des dienstlichen Dokuments, des Symbols oder einer Marke auszuarbeiten.

Nach dem Art. 1264 des BGB sind Entwürfe von offiziellen Dokumenten, einschließlich der Übersetzungen von Dokumenten, sowie Symbolen- und Markenprojekte durch das Urheberrecht geschützt und da entstehen Rechte auf geistiges Eigentum. Im Zivilrecht wird die Möglichkeit nicht vorgesehen, den obenerwähnten Objekten den Status eines dienstlichen Produktes der intellektuellen Tätigkeit zu verleihen.

Es wurde versucht, dieses Dilemma in wissenschaftlichen Kreisen zu lösen. Einer der Forscher dieses Problems ist P.P. Battahov, der es für zweckmäßig hält, den Art. 1264 des BGB zu ändern, und zwar auf folgende Weise: "Wenn das Projekt eines offiziellen Dokuments, des Symbols oder der Marke ein dienstliches Produkt ist, so besitzt der Arbeitgeber das Recht, bestimmte Handlungen für dessen Bekanntmachung damit durchzuführen, er hat auch das Recht, dem Produkt einen Namen zu geben. Der Autor des offiziellen Projekts und Symbols oder der Marke behält auch das Recht auf Vergütung ... Der Entwickler des Projekts von einem offiziellen Dokument, Symbol oder einer Marke ist die Person, die das Projekt erarbeitet hat, d. h. sein Arbeitgeber..." [1]

Offizielle Dokumente - sind die, "die von den Organen der gesetzgebenden, vollziehenden und rechtsprechenden Gewalten angenommen sind und die einen obligatorischen, empfehlenden oder informativen Charakter tragen" 3 .

Nehmen wir an, dass eine ArbeitgeberOrganisation in der Entwicklung von offiziellen Dokumentprojekten u.ä. spezialisiert ist. In den 
Vorschriften des Art. 1264 des BGB ist festgelegt, dass das Projekt nur auf Antrag einer staatlichen oder einer kommunalen Behörde sowie einer internationalen Organisation entwickelt werden darf.

Gemäß den Bestimmungen des Art. 1288 des BGB wird im Autorenvertrag vorausgesetzt, dass der Autor für den Auftraggeber ein vereinbartes Produkt schafft. Allerdings wird dieses Ergebmis der intellektuellen Tätigkeit offiziell nicht anerkannt.

Entsprechend dem Art. 1298 des BGB können Werke aus dem Bereich der Wissenschaft, Literatur und Kunst nur durch den staatlichen oder kommunalen Vertrag geschaffen werden, in diesem Fall gehören die Rechte (ausschließliche Rechte) auf dieses Ergebmis der intellektuellen Tätigkeit dem Vollzieher oder Darsteller, d.h. dem Autor, oder einer anderen Person, die den staatlichen oder kommunalen Auftrag ausführt (einschließlich des Auftrags einer Organisation, der Autor angehört).

Es sei bemerkt, dass die Bestimmungen des Art. 1264 und des Art. 1298 des BGB nach ihren subjektiven Tatbeständen nicht zusannebfallen. Der Projektsentwickler ist eine Person, die das entsprechende Projekt erarbeitet hat. Der Gesetzgeber bezeichnet ihn nicht "als den Autor des Projekts" und gibt keinen Hinweis daauf, ob das eine natürliche oder juristische Person ist. Aber im Wortlaut des Art. 1264 gibt es einen Hinweis auf den "Namen", der nur auf eine natürliche Person angewendet werden kann, die als Entwickler auftritt. Juristische Person hat seine "Bezeichnung" (Art. 54 des BGB der RF, der "Name" ist aber eine der individualisierenden Eigenschaften eben einer natürlichen Person.

Wenn wir annehmen, dass die juristische Person als Arbeitgeber für den Entwickler-Autor fungiert, dann ist es wirklich notwendig, die Bestimmungen des Art. 1264 des BGB zu korrektieren und sie mit dem Art. 1295 des BGB der RF in Übereinstimmung zu bringen. Das bedeutet jedoch, dass der Autor schon eine unmittelbare Verantwortung übernimmt, bei der Erfüllung seiner Arbeitsaufgaben ein solches dienstliches Produkt zu schaffen, dabei sollen die Ergebnisse der kreativen Tätigkeit in der jeweiligen Organisation auch anwendbar sein. Daher ist es schwierig, sich die Umsetzung der Vorschriften über dienstliches Projekt eines offiziellen Dokumentes vorzustellen.
In dieser Hinsicht scheint die Frage nach dem Rechtsschutz eines "Projektes" als Grundlage für ein dienstliches Produkt am interessanten zu sein.

In der juristischen Literatur wird ein anderes Verständnis von geistigem Eigentum angeboten. A. Dozortsev zählt eine Anzahl von Merkmalen, die kreative Ergebnisse charakterisieren, und zwar: a) ihren inmateriellen Charakter, b) kommerziellen Wert, c) ästhetischen oder informativen Inhalt, g) die Möglichkeit der Absonderung der Ergebnisse von anderen Objekten [4].

Alle diese Eigenschaften kann auch das "Projekt" eines dienstlichen Ergebnisses der intellektuellen Tätigkeit (insbesondere kommerziellen Wert) besitzen, aber dabei kann es kein Objekt der intellektuellen im klassischen Sinne sein.

Wenn ein Mitarbeiter entlassen wird, nachdem er ein Objekt der geistigen Eigentumsrechte geschaffen hat und sein Anteil zum Beispiel dabei nur $90 \%$ beträgt, so bleibt das Schicksal seiner früheren bei der Erfüllung seiner Arbeitspflichten gemachten Entwicklungen nicht bestimmt.

Der Rechtsschutz des "Projektes" eines offiziellen Ergebnisses oder Produkts der intellektuellen Tätigkeit und die Verankerung der Rechte des Arbeitnehmers und des Arbeitgebers würden die Interessen beider Seiten beachten. Dies würde dazu beitragen, diese Lücke in der gesetzlichen Regelung der Rechtsbeziehungen im Bereich der Schaffung und Nutzung des kreativen Ergebnisses auszufüllen.

Dieses "Projekt" soll individuell sein und die Merkmale eines konkreten, rechtlich geschützten Ergebnisses der geistigen Tätigkeit besitzen.

Dem Arbeitgeber muss das Recht auf "Projekt" eines dienstlichen Objektes des geistigen Eigentums, einschließlich aller Materialie und Entwicklungen, gewährt werden. Es würde in diesem Fall die Möglichkeit erhalten, es für die künftige Schaffung von einem schützbaren kreativen Produkt zu verwenden. Wenn auf der Grundlage dieses "Projekts" ein solches Ergebnis durch einen anderen Arbeitnehmer geschaffen wird, so wird der Entwickler des "Projekts" (auch nach seinen Ausscheiden aus der Organisation) die Rechte des Mitautors erwerben sowie ein Recht auf Vergütung.

Es wäre auch zweckmäßig, dem Arbeitgeber das Recht zu geben, ein "Projekt" 
als Geheimnis zu bewahren. Wenn ein Objekt der intellektuellen Rechte nach einer bestimmten Zeit nicht geschaffen wird oder das Regime des kommerziellen Geheimnisses nicht eingeführt wird, so blebt seinem Entwickler das Recht erhalten, dieses "Projekt" für die Schaffung eines kreativen Ergebnisses selbstsändig zu verwenden (wenn er auch seine Arbeitsfunktionen schon für einen anderen Arbeitgeber ausführt).

Wie bereits erwähnt wurde, gehört ein Objekt der intellektuellen Rechte zum Eigentum der Organisation, da sie einen großen Umfang von Befugnissen hat. In der russischen Gesetzgebung fehlt jedoch kein klares Verständnis von dem "dienstlichen Ergebnis der intellektuellen Tätigkeit”, auf das der Arbeitgeber diese oder jene Rechte hat.

Die Wissenschaftler bieten ihre Definitionen der genannten Rechtskategorie. E.V. Gaboyan äußert die Meinung, unter einem dienstlichen Produkt sei ein in eine objektive Form gehülltes inmaterielles Ergebnis der kreatuven Arbeit des Arbeitnehmers zu verstehen, das im Rahmen seiner Arbeitsverhältnisse mit dem Arbeitgeber geschaffen worden ist. Als "das einzige Kriterium der Zuordnung des Produkts zu den dienstlichen Produkten gilt "die Schaffunng eines Objekts des Urheberrechts im Rahmen der beruflichen Pflichten" des Arbeitsnehmers [2], die im Vertrag, was in der Stellenbeschreibung oder in einer Anirdnung festgelegt sind.

P.P. Battahov bietet $\mathrm{n}$ seiner Forschung folgende Definition an: "Das dienstliche Objekts der intellektuellen Rechte ist das geschützte Ergebnis der intellektuallen Tätigkeit oder ein Produktionsgehimnis (Know-how)", das bei der Erfüllung seiner Arbeitspflicht von einem Arbeitgeber im Rahmen seiner vertraglich festgelegten Funktionen geschaffen ist [1]. Der Autor verweist darauf, dass das Geheimnis der Produktion eine "besondere Aufgabe" ist, und das erlaubt nicht, die Normen der Ergebnisse der intellektuellen Tätigkeit auf dieses Objekt anzuwenden. Dieser von dem Forscher definierte Begrifff entspricht den geltenden Rechtsvorschriften nicht, und P.P. Battahov schließt das Know-how aus der Anzahl des geschützten geistigen Eigentums aus.

V.O. Dobrynin, der die Besonderheiten der rechtlichen Regulierung von Erfindungen in der Gesetzgebung der Russischen Föderation erforscht hat (sowohl im Ausland als auch in der RF), bietet auch eine Definition des dienstliches Ergebnisses der intelektuellen Tätigkeit an. Er versteht unter einem komkreten Auftrag des Arbeitsgebers den Auftrag, den er innerhalb oder außerhalb seiner Arbeitspflichten vom Arbeitgeber zur Ausführung erhält, der einen bestimmten Zweck verfolgt und Informationen über die Ausführungszeit enthält sowie von ihm die Ausübung der intellektuellen Tätigkeit erfordert... [3].

Was die Definition des Begriffes des Auftrags im Teil der Ausführung der Arbeit "außerhalb der Arbeitpflichten" angeht, so könnte man sich mit dem Autor deswegen auseinandersetzen. Das Arbeitsgesetzbuch der RF verbietet, von einem Arbeitnehmer die im Arbeitsvertrag nicht vorgesehenen Arbeitsleistungen zu fordern.

Aoßerdem verweist Dobrynin darauf, dass man auf eine Erfindung ein bestimmtes, d.h. dienstliches Kriterium angelegt werden muss, das den "Tätigkeitsbereich des Arbeitgebers" angibt. Das wäre eine durchaus sinnvolle und praxisgerichtete Präzisierung.

Es sei bemerkt, dass die russische Gesetzgebung keine angemessene gesetzliche Regelung der Schaffung eines von staatlichen und kommunalen Bediensteten geschaffenen dienstlichen Objekts des geistigen Eigentums. Es besteht jedoch für die genannten Arbeitnehmer potenziale Möglichkeit, so ein Produkt zu schaffen.

\section{Schlussfolgerungen}

Mit Rücksicht auf das Obengesagte können wir folgende Schlußfolgerung ziehen: Es ist notwendig, alle gestzlichen Vorschriften über dienstliche Produkte der intellektuellen Tätigkeit als ein einheitliches System aufzubauen. Das BGB der RF soll allgemeine Bestimmungen enthalten, die den Begriff des dienstlichen Produkts des geistigen Eigentums verankert.

Das dienstliche Product der intellektuellen Tätigkeit ist ein Objekt der intellektuellen Rechte, das als Ergebnis im Laufe der Ausführung der Arbeitspflichten sowie eines konkreten Auftrags des Arbeitsgebers von einem Autor, Träger der Arbeits- oder Dienstverhältnisse, geschaffen ist, das als Objekt der intellektuellen Rechte geschützt ist und das den Kriterien der Anwendbarkeit im Tätigkeitsfeld des Aebeitsgebers entspricht. 


\section{FUßNOTE}

${ }^{1}$ Über das kommerzielle Geheimniss s. das Bundesgesetz der Russischen Föderation vom 29. Juli 2004 N 98 (Ausgabe vom 2014.12.03), angenommen von der Staatsduma der Bundesversammlung der RF am 9. Juli 2004 genehmigt vom Föderationsrat ym 15. Juli 2004 // Die russische Zeitung. 2004. 5. August.

2 In Bezug auf die Umsetzung der Leitlinien für die Prüfung der Anträge auf Erfindungen: des Befehls von Rospatent am 25. Juli 2011 N 87 (Ausgabe von 2014.01.14). URL: http://www1.fips.ru/wps/wcm/ connect/content_ru/ru/inventions_utility_models/ ruk_ezp_iz (Stichtag: 25/11/2015).

${ }^{3}$ Über eine obligatorische Kopie der Dokumente: Bundesgesetz der Russischen Föderation vom 29. Dezember. 1994 N 77 (Ausgabe vom 2014.05.05), angenommen von der Staatsduma, der Bundesversammlung der Russischen Föderation am 23. November 1994 // Die russische Zeitung. 1995. 17. Januar.

\section{REFERENCES}

1. Battakhov P.P. Sluzhebnye rezultaty intellektualnoy deyatelnosti po zakonodatelstvu Rossiyskoy Federatsii: avtoref. dis. ... kand. yurid. nauk [Employment-Related Results of Activity According to the Legislation of the Russian Federation. Cand. jurid. sci. abs. diss.]. Moscow, 2010. $29 \mathrm{p}$.

2. Gaboyan E.P. Pravovoe regulirovanie otnosheniy, voznikayushchikh $v$ svyazi s sozdaniem $i$ ispolzovaniem sluzhebnykh obyektov avtorskogo $i$ patentnogo prava: avtoref. dis. ... kand. yurid. nauk [Legal Regulation of Relations Established due to the Use of Employment-Related Objects of Author's and Patent Rights. Cand. jurid. sci. abs. diss.]. Available at: http://dissovet.rggu.ru/binary/object_1.1321360 750.04229.doc (accessed April 15, 2016).

3. Dobrynin V.O. Osobennosti pravovogo regulirovaniya sluzhebnykh izobreteniy: dis. ... kand. yurid. nauk [Features of Legal Regulation of Employment-Related Inventions]. Available at: http:// dis.rgiis.ru/files/dis/d40100102/dobrynin-051214/ dobrynin-051214-d.pdf(accessed April 16, 2016).

4. Dozortsev V.A. Intellektualnye prava: Ponyatie. Sistema. Zadachi kodifikatsii [Intellectual Rights: Concept. System. Codification Tasks]. Moscow, Statut Publ., 2005. 416 p.

5. Krupko S. Materialno-pravovye aspekty izobreteniy rabotnikov [Material and Legal Aspects of Employees' Inventions]. Khozyaystvo i pravo, 2011, no. 8, pp. 3-30.

\section{Information About the Author}

Olga V. Nikulina, Postgraduate Student, the Department of Civil Law Disciplines, Volgograd Institute of Management - the Branch of the Russian Academy of National Economy and Public Administration under the President of the Russian Federation, Gagarina St., 8, 400131 Volgograd, Russian Federation, yamboglo89@mail.ru.

\section{Информация об авторе}

Ольга Владимировна Никулина, аспирант кафедры гражданско-правовых дисциплин, Волгоградский институт управления - филиал Российской академии народного хозяйства и государственной службы при Президенте РФ, ул. Гагарина, 8, 400131 г. Волгоград, Российская Федерация, yamboglo89@mail.ru.

\section{Informationen über den Autor}

Olga W. Nikulina, Jungakademiker, Lehrstuhl für Staats- und Zivilrechtswissenschaften, Russische Akademie der Volkswirtschaft und öffentliche Verwaltung beim Präsidenten der Russischen Föderation, Str. Gagarin, 8, 400131 Wolgograd, Russischen Föderation, yamboglo89@mail.ru. 